\title{
RESULTS ON COMMON FIXED POINTS
}

\author{
ZEQING LIU and JEONG SHEOK UME
}

(Received 9 October 2000)

\begin{abstract}
We establish common fixed point theorems related with families of selfmappings on metric spaces. Our results extend, improve, and unify the results due to Fisher (1977, 1978, 1979, 1981, 1984), Jungck (1988), and Ohta and Nikaido (1994).
\end{abstract}

2000 Mathematics Subject Classification. 47H10, 54H25.

1. Introduction. Let $w$ and $\mathbb{N}$ denote the sets of nonnegative integers and positive integers, respectively. For $t \in[0, \infty),[t]$ denotes the largest integer not exceeding $t$. Let $f$ and $g$ be mappings from a metric space $(X, d)$ into itself and $C_{f}=\{h: h: X \rightarrow$ $X$ and $h f=f h\}$. For $x, y \in X$ and $A \subseteq X$, define $O_{f}(x)=\left\{f^{n} x: n \in w\right\}, O_{f}(x, y)=$ $O_{f}(x) \cup O_{f}(y), O_{f, g}(x)=\left\{f^{i} g^{j} x: i, j \in w\right\}, O_{f, g}(x, y)=O_{f, g}(x) \cup O_{f, g}(y)$, and $\delta(A)=\sup \{d(x, y): x, y \in A\}$. Let $i_{X}$ denote the identity mapping on $X$. It is easy to see that $\left\{f^{n}: n \in w\right\} \subseteq C_{f}$. Let $\Phi=\{\varphi: \varphi:[0, \infty) \rightarrow[0, \infty)$ is upper semicontinuous and nondecreasing and $\varphi(t)<t$ for $t>0\}$. The following definition and lemmas were introduced by Fisher [8], Singh and Meade [13], and Jungck [10], respectively.

Definition 1.1 (see [8]). Let $A \subseteq X$ and $A_{n} \subseteq X$ for all $n \in \mathbb{N}$. The sequence $\left\{A_{n}\right\}_{n \in \mathbb{N}}$ is said to converge to $A$ if

(i) each point $a \in A$ is the limit of some convergent sequence $\left\{a_{n}\right\}_{n \in \mathbb{N}}$, where $a_{n} \in A_{n}$ for all $n \in \mathbb{N}$;

(ii) for arbitrary $\varepsilon>0$, there exists an integer $k$ such that $A_{n} \subseteq A_{\varepsilon}$ for $n>k$, where $A_{\varepsilon}$ is the union of all open spheres with centers in $A$ and radius $\varepsilon$.

LEMMA 1.2 (see [13]). Let $\varphi \in \Phi$, then (a) $\lim _{n \rightarrow \infty} \varphi^{n}(t)=0$ for all $t>0$ and (b) $t=0$ provided that $t \leq \varphi(t)$ for some $t \geq 0$.

LEMMA 1.3 (see [10]). Let $f$ and $g$ be commuting self-mappings of a compact metric space $(X, d)$ such that $g f$ is continuous. If $A=\bigcap_{n \in \mathbb{N}}(g f)^{n} X$, then

(i) $h A \subseteq A$ for all $h \in C_{g f}$;

(ii) $A=f A=g A \neq \phi$;

(iii) $A$ is compact.

In recent years, a number of generalizations of a well-known contraction mapping principles due to Banach have appeared in the literature (cf. [1, 2, 3, 4, 5, 6, 7, 8, 9, 10, $11,12,13])$. 
First we list the following general conditions.

(1) There exists $\varphi \in \Phi$ and $p, q, m, n \in w$ with $p+q, m+n \in \mathbb{N}$ such that

$$
d\left(f^{p} g^{q} x, f^{m} g^{n} y\right) \leq \varphi\left(\delta\left(\bigcup_{h \in C_{g} \cup C_{f}} h O_{g, f}(x, y)\right)\right)
$$

for all $x, y \in X$.

(2) There exist $p, q, m, n \in w$ with $p+q, m+n \in \mathbb{N}$ such that

$$
d\left(f^{p} g^{q} x, f^{m} g^{n} y\right)<\delta\left(\bigcup_{h \in C_{g f}} h O_{g f}(x, y)\right)
$$

for all $x, y \in X$ with $f^{p} g^{q} x \neq f^{m} g^{n} y$.

In the literature of fixed point theory there exist conditions which are special cases of (1) or (2). Now we list below some of the contractive mappings for which various fixed point theorems have been established.

(3) (See [6].) There exists $r \in[0,1)$ and $p, q, m, n \in w$ with $p+q, m+n \in \mathbb{N}$ such that

$$
\begin{aligned}
& d\left(f^{p} g^{q} x, f^{m} g^{n} y\right) \\
& \leq r \cdot \max \left\{d\left(f^{r} g^{r^{\prime}} x, f^{s} g^{s^{\prime}} y\right),\right. \\
& \quad d\left(f^{r} g^{r^{\prime}} x, f^{t} g^{t^{\prime}} x\right), d\left(f^{s} g^{s^{\prime}} x, f^{i} g^{i^{\prime}} y\right): \\
& \left.\quad 0 \leq r, t \leq p, 0 \leq r^{\prime}, t^{\prime} \leq q, 0 \leq s, i \leq m, 0 \leq s^{\prime}, i^{\prime} \leq n\right\}
\end{aligned}
$$

for all $x, y \in X$.

(4) (See [6].) There exist $p, q, m, n \in w$ with $p+q, m+n \in \mathbb{N}$ such that

$$
\begin{aligned}
& d\left(f^{p} g^{q} x, f^{m} g^{n} y\right) \\
& <\max \left\{d\left(f^{r} g^{r^{\prime}} x, f^{s} g^{s^{\prime}} y\right),\right. \\
& d\left(f^{r} g^{r^{\prime}} x, f^{t} g^{t^{\prime}} x\right), d\left(f^{s} g^{s^{\prime}} x, f^{i} g^{i^{\prime}} y\right): \\
& \left.0 \leq r, t \leq p, 0 \leq r^{\prime}, t^{\prime} \leq q, 0 \leq s, i \leq m, 0 \leq s^{\prime}, i^{\prime} \leq n\right\}
\end{aligned}
$$

for all $x, y \in X$ for which the right-hand side of the inequality is positive.

(5) (See [11].) There exist $k \in \mathbb{N}$ and $r \in[0,1)$ such that

$$
d\left(f^{k} x, f^{k} y\right) \leq r \delta\left(O_{f}(x, y)\right)
$$

for all $x, y \in X$.

(6) (See [10].) For all $x, y \in X, d(f x, g y)<\delta\left(h t: t \in\{x, y\}, h \in C_{g f}\right)$ with $f x \neq g y$.

In this note, we prove common fixed point theorems for $C_{f} \cap C_{g}$ and $C_{g f}$ in bounded complete metric spaces and compact metric spaces. Our results extend, improve and unify the corresponding results in $[1,2,3,4,5,6,7,9,10,11]$.

2. Common fixed point theorems. Our main results are as follows.

THEOREM 2.1. Let $f$ and $g$ be commuting mappings from a bounded complete metric space $(X, d)$ into itself. If $f$ and $g$ are continuous and satisfy (1), then

(i) $f$ and $g$ have a unique common fixed point $u \in X$ which is also a unique common fixed point of $C_{f} \cap C_{g}$; 
(ii) $d\left(f^{i+a} g^{i+b} x, u\right) \leq \varphi^{[i / k]}(\delta(X))$ for all $a, b \in\{0,1\}$ and $i \in \mathbb{N}$, where $k=$ $\max \{p, q, m, n\}$;

(iii) $\lim _{i \rightarrow \infty} f^{i+a} g^{i+b} x=u$ for all $a, b \in\{0,1\}$;

(iv) $\left\{f^{i} g^{i} X\right\}_{i \in \mathbb{N}}$ converges to $\{u\}$.

Proof. For any $i \in w$ and $x, y \in X$, it follows from (1) that

$$
\begin{aligned}
& d\left(f^{i+k} g^{i+k} x, f^{i+k} g^{i+k} y\right) \\
& \quad \leq \varphi\left(\delta\left(\bigcup_{h \in C_{f} \cap C_{g}} h O_{f, g}\left(f^{i+k-p} g^{i+k-q} x, f^{i+k-m} g^{i+k-n} y\right)\right)\right) \\
& \quad \leq \varphi\left(\delta\left(\bigcup_{h \in C_{f} \cap C_{g}} h O_{f, g}\left(f^{i} g^{i} x, f^{i} g^{i} y\right)\right)\right) \\
& \quad \leq \varphi\left(\delta\left(\bigcup_{h \in C_{f} \cap C_{g}} h f^{i} g^{i} X\right)\right) \\
& \quad=\varphi\left(\delta\left(\bigcup_{h \in C_{f} \cap C_{g}} f^{i} g^{i} h X\right)\right) \\
& \quad=\varphi\left(\delta\left(f^{i} g^{i} X\right)\right)
\end{aligned}
$$

which implies that

$$
\delta\left(f^{i+k} g^{i+k} X\right)=\sup _{x, y \in X} d\left(f^{i+k} g^{i+k} x, f^{i+k} g^{i+k} y\right) \leq \varphi\left(\delta\left(f^{i} g^{i} X\right)\right)
$$

for all $i \in w$. We can write $i=j k+t$ uniquely for some $j, k \in w$ with $t<k$. Thus

$$
\delta\left(f^{i} g^{i} X\right) \leq \varphi\left(\delta\left(f^{(j-1) k+t} g^{(j-1) k+t} X\right)\right) \leq \varphi^{j}\left(\delta\left(f^{t} g^{t} X\right)\right) \leq \varphi^{j}(\delta(X)) .
$$

It follows from the boundedness of $X$, Lemma 1.2 and (2.3) that

$$
\lim _{i \rightarrow \infty} \delta\left(f^{i} g^{i} X\right)=\lim _{j \rightarrow \infty} \varphi^{j}(\delta(X)) .
$$

Since $d\left(f^{i} g^{i} x, f^{i+t} g^{i+t} x\right) \leq \delta\left(f^{i} g^{i} X\right)$ for all $i, t \in w$ and $x \in X,\left\{f^{i} g^{i} x\right\}_{i \in \mathbb{N}}$ is a Cauchy sequence and converges to some $u \in X$ by completeness of $X$. For any $i, c \in w$, $a, b \in\{0,1\}$ and $x \in X$, by (2.3) and (2.4) we have

$$
d\left(f^{i+a} g^{i+b} x, f^{i+c} g^{i+c} x\right) \leq \delta\left(f^{i} g^{i} X\right) \leq \varphi^{[i / k]}(\delta(X)) .
$$

Letting $c$ tend to infinity, we get

$$
d\left(f^{i+a} g^{i+b} x, u\right) \leq \varphi^{[i / k]}(\delta(X))
$$

for $x \in X$. The continuity of $f$ and $g$, and (2.4) and (2.6) ensure that

$$
u=\lim _{i \rightarrow \infty} f^{i} g^{i} x=\lim _{i \rightarrow \infty} f^{a} g^{b} f^{i} g^{i} x=f^{a} g^{b} u
$$

for all $a, b \in\{0,1\}$ and $x \in X$. That is, $f u=g u=u$. 
Suppose that $f$ and $g$ have another common fixed point $v \in X$. It follows from (2.4) that

$$
d(u, v) \leq \delta\left(f^{i} g^{i} X\right) \leq \varphi^{[i / k]}(\delta(X)) \longrightarrow 0 \quad \text { as } i \longrightarrow \infty .
$$

That is, $u=v$. Therefore $f$ and $g$ have a unique common fixed point. Note that $f h u=$ $h f u=h u=h g u=g h u$ for all $h \in C_{f} \cap C_{g}$. By the uniqueness of common fixed point of $f$ and $g$, we have $h u=u$ for all $h \in C_{f} \cap C_{g}$. Since $f, g \in C_{f} \cap C_{g}$, it follows that $u$ is a unique common fixed point of $C_{f} \cap C_{g}$.

Let $\varepsilon>0$. In view of (2.4), there exists $c \in \mathbb{N}$ such that

$$
\delta\left(f^{i} g^{i} X\right) \leq \varphi^{[i / k]}(\delta(X))<\frac{1}{2} \varepsilon .
$$

for all $i>c$. Note that $u \in f^{i} g^{i} X$ for all $i \in w$. Thus, for any $i>c$ we have

$$
f^{i} g^{i} X \subseteq B(u, \varepsilon)=\{x \in X: d(u, x)<\varepsilon\} .
$$

Therefore $\left\{f^{i} g^{i} X\right\}_{i \in \mathbb{N}}$ converges to $\{u\}$. This completes the proof.

REMARK 2.2. [6, Theorem 2] is a special case of Theorem 2.1.

As an immediate consequence of Theorem 2.1, we have the following corollary.

COROLLARY 2.3. Let $f$ and $g$ be commuting mappings from a bounded complete metric space $(X, d)$ into itself. If $f$ and $g$ are continuous and satisfy the inequality

$$
d\left(f^{p} g^{q} x, f^{m} g^{n} y\right) \leq r \delta\left(\bigcup_{h \in C_{f} \cap C_{g}} h O_{f, g}(x, y)\right)
$$

for all $x, y \in X$, where $p, q, m, n \in w, p+q, m+n \in \mathbb{N}$ and $r \in[0,1)$. Then (i), (iii), and (iv) of Theorem 2.1 and the following ( $v$ ) hold:

(v) $d\left(f^{i+a} g^{i+b} x, u\right) \leq r^{[i / k]} \delta(X)$ for all $a, b \in\{0,1\}$ and $i \in \mathbb{N}$, where $k=$ $\max \{p, q, m, n\}$.

REMARK 2.4. In case $p=m, g=i_{X}$, Corollary 2.3 reduces to a result which generalizes [11, Theorem 3].

THEOREM 2.5. Let $f$ and $g$ be commuting mappings from a compact metric space $(X, d)$ into itself such that $g f$ is continuous. If (2) is satisfied, then $f$ and $g$ have a unique common fixed point $u \in X$. Moreover, $u=h u$ for all $h \in C_{g f}$.

Proof. Let $A=\bigcap_{n \in \mathbb{N}}(g f)^{n} X$. It follows from Lemma 1.3 that $A=f A=g A \neq \varnothing$ and that $A$ is compact. We claim that $A=\{u\}$ for some $u \in X$. Otherwise $\delta(A)>0$. By the compactness of $A$ there exists distinct $u, v \in A$ such that $\delta(A)=d(u, v)$. Clearly, we can find $x, y \in A$ such that $f^{p} g^{q} x=u$ and $f^{m} g^{n} y=v$. Using (2) and Lemma 1.3 we have

$$
\delta(A)=d\left(f^{p} g^{q} x, f^{m} g^{n} y\right)<\delta\left(\bigcup_{h \in C_{g f}} h O_{g f}(x, y)\right) \leq \delta\left(\bigcup_{h \in C_{g f}} h A\right) \leq \delta(A)
$$

which is a contradiction. Thus $A=\{u\}$ for some $u \in X$. Lemma 1.3 ensures that $u=h u$ for all $h \in C_{g f}$. In particular, $u=f u=g u$. If $f$ and $g$ have another common fixed point $c \in X$. Then $c=(g f)^{n} c$ for all $n \in \mathbb{N}$. That is, $c \in A=\{u\}$. Hence $u$ is the only common fixed point of $f$ and $g$. This completes the proof. 
REMARK 2.6. Theorem 2.5 includes [6, Theorem 5] as a special case.

As an immediate consequence of Theorem 2.5, we have the following corollary.

COROLLARY 2.7. Let $f$ and $g$ be commuting mappings from a compact metric space $(X, d)$ into itself. If $g f$ is continuous and there exists $p, m \in \mathbb{N}$ such that

$$
d\left(f^{p} x, g^{m} y\right)<\delta\left(\bigcup_{h \in C_{g f}} h O_{g f}(x, y)\right)
$$

for all $x, y \in X$ with $f^{p} x \neq g^{m} y$. Then the conclusion of Theorem 2.5 holds.

COROLLARY 2.8. Let $f$ be a continuous mapping from a compact metric space $(X, d)$ into itself. Assume that there exists $p, m \in \mathbb{N}$ such that

$$
d\left(f^{p} x, f^{m} y\right)<\delta\left(\bigcup_{h \in C_{f}} h O_{f}(x, y)\right)
$$

for all $x, y \in X$ with $f^{p} x \neq f^{m} y$. Then $f$ has a unique fixed point $u \in X$ and $h u=u$ for all $h \in C_{f}$.

REMARK 2.9. Corollary 2.7 extends, improve and unifies [10, Theorem 4.2], [9, Theorem 2], and [7, Theorem 5].

REMARK 2.10. [1, Theorem 4], [2, Theorem 4], [4, Theorem 9], [3, Theorem 2], and [5, Theorem 4] are special cases of Corollary 2.8.

REMARK 2.11. The following examples reveal that the condition that $f$ and $g$ are continuous is necessary in Theorems 2.1, 2.5, and Corollaries 2.7, 2.8.

EXAMPLE 2.12. Let $X=[0,1]$ with the usual metric $d$. Define $f, g: X \rightarrow X$ by $g=i_{X}$, $f 0=1 / 4$ and $f x=(1 / 3) x$ for all $X \in(0,1]$. Take $p=m=2, q=n=1, r=1 / 2$, $\varphi(t)=(1 / 2) t$ for all $t \geq 0$. Then $(X, d)$ is a bounded metric space, $g$ is continuous, and $f$ is discontinuous.

For $x, y \in(0,1]$, we have

$$
\begin{aligned}
d\left(f^{p} g^{q} x, f^{m} g^{n} y\right) & =\frac{1}{9}|x-y| \\
& \leq \frac{1}{2}|x-y| \leq \frac{1}{2} \delta\left(O_{f, g}(x, y)\right) \\
& \leq \varphi\left(\delta\left(\bigcup_{h \in C_{f} \cap C_{g}} h O_{f, g}(x, y)\right)\right) .
\end{aligned}
$$

For $x=0, y \in(0,1]$ or $x \in(0,1], y=0$, we have

$$
\begin{aligned}
d\left(f^{p} g^{q} x, f^{m} g^{n} y\right) & \leq \max \left\{\left|\frac{1}{12}-\frac{1}{9} y\right|,\left|\frac{1}{12}-\frac{1}{9} x\right|\right\}<\frac{1}{8} \\
& =\frac{1}{2} \delta\left(O_{f, g}(0)\right) \leq \varphi\left(\delta\left(\bigcup_{h \in C_{f} \cap C_{g}} h O_{f, g}(x, y)\right)\right) .
\end{aligned}
$$


For $x=y=0$, we have

$$
d\left(f^{p} g^{q} x, f^{m} g^{n} y\right)=0<\frac{1}{8}=\frac{1}{2} \delta\left(O_{f, g}(0,0)\right) \leq \varphi\left(\delta\left(\bigcup_{h \in C_{f} \cap C_{g}} h O_{f, g}(x, y)\right)\right)
$$

That is, $f$ and $g$ satisfy (1) and (2.11). But $f$ and $g$ have no common fixed point in $X$.

EXAMPLE 2.13. Let $(X, d), f, g, p, q, m$, and $n$ be as in Example 2.12. It is easy to check that the conditions of Theorem 2.5 and Corollary 2.8 are satisfied except for the continuity assumption. However $f$ has no fixed point in $X$.

ACKNOWLEDGEMENT. The second author was financially supported by Changwon National University in 2000.

\section{REFERENCES}

[1] B. Fisher, On three fixed point mappings for compact metric spaces, Indian J. Pure Appl. Math. 8 (1977), no. 4, 479-481. MR 57\#7562. Zbl 366.54026.

[2] _ Some theorems on fixed points, Studia Sci. Math. Hungar. 12 (1977), no. 1-2, 159160. MR 81j:54065. Zbl 434.54034.

[3] _ A fixed-point theorem for compact metric spaces, Publ. Math. Debrecen 25 (1978), no. 3-4, 193-194. MR 80c:54054. Zbl 389.54030.

[4] _ Theorems on fixed points, Riv. Mat. Univ. Parma (4) 4 (1978), 109-114. MR 80i:54054. Zbl 404.54043.

[5] _ Quasicontractions on metric spaces, Proc. Amer. Math. Soc. 75 (1979), no. 2, $321-$ 325. MR 80m:54058. Zbl 411.54049.

[6] _ Results on common fixed points on bounded metric spaces, Math. Sem. Notes Kobe Univ. 7 (1979), no. 1, 73-80. MR 80h:54057. Zbl 415.54029.

[7]_ Common fixed points of commuting mappings, Bull. Inst. Math. Acad. Sinica 9 (1981), no. 3, 399-406. MR 83a:54066. Zbl 466.54037.

[8] _ Common fixed points of mappings and set-valued mappings, Rostock. Math. Kolloq. (1981), no. 18, 69-77. MR 83e:54041. Zbl 479.54025.

[9]_ A common fixed point theorem for four mappings on a compact metric space, Bull. Inst. Math. Acad. Sinica 12 (1984), no. 3, 249-252. MR 85m:54047. Zbl 544.54037.

[10] G. Jungck, Common fixed points for commuting and compatible maps on compacta, Proc. Amer. Math. Soc. 103 (1988), no. 3, 977-983. MR 89h:54030. Zbl 661.54043.

[11] M. Ohta and G. Nikaido, Remarks on fixed point theorems in complete metric spaces, Math. Japon. 39 (1994), no. 2, 287-290. MR 94m:54099. Zbl 802.47055.

[12] B. E. Rhoades, A comparison of various definitions of contractive mappings, Trans. Amer. Math. Soc. 226 (1977), 257-290. MR 55\#6406. Zbl 365.54023.

[13] S. P. Singh and B. A. Meade, On common fixed point theorems, Bull. Austral. Math. Soc. 16 (1977), no. 1, 49-53. MR 55\#11234. Zbl 351.54040.

Zeqing LiU: Department of MAThematics, LiaOning Normal University, Dalian, LIAONING 116029, CHINA

Jeong Sheok Ume: Department of ApPlied Mathematics, Changwon National UniverSITY, CHANGWON 641-773, KOREA

E-mail address: j sume@sarim. changwon.ac. kr 


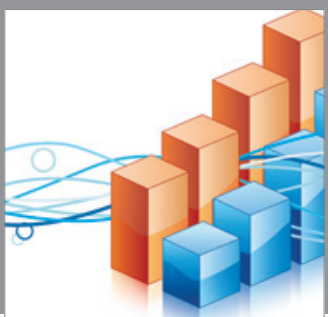

Advances in

Operations Research

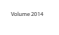

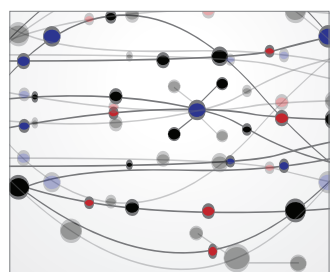

\section{The Scientific} World Journal
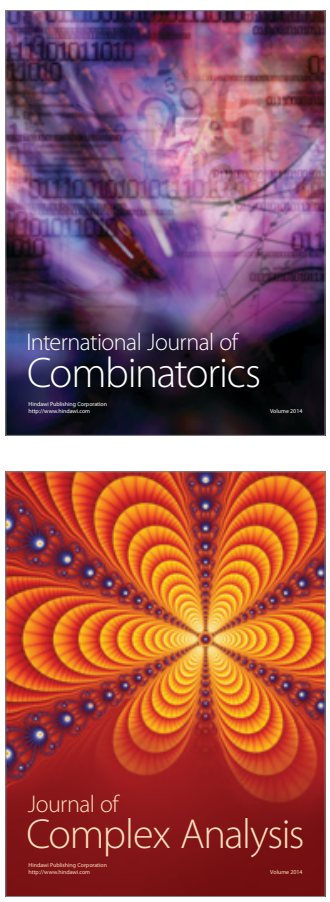

International Journal of

Mathematics and

Mathematical

Sciences
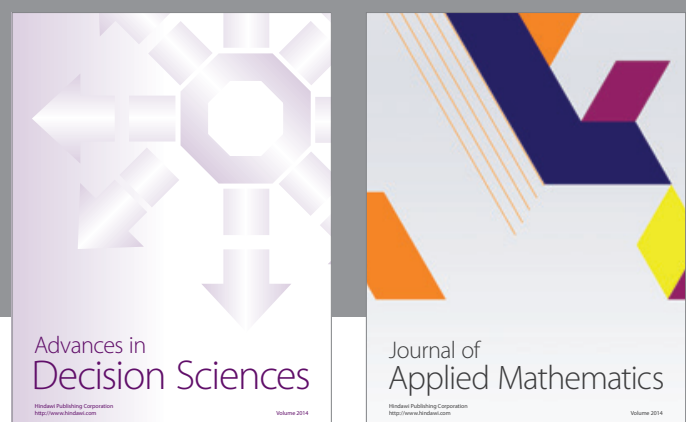

Journal of

Applied Mathematics
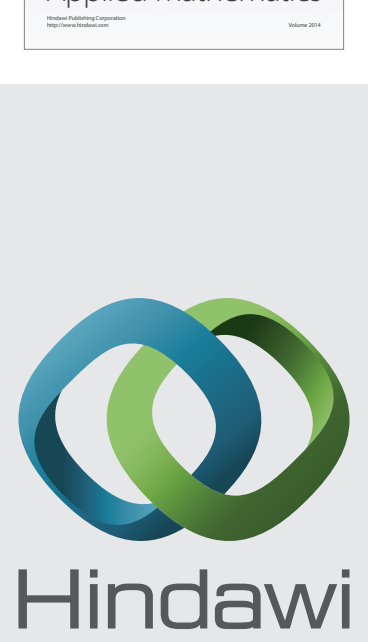

Submit your manuscripts at http://www.hindawi.com
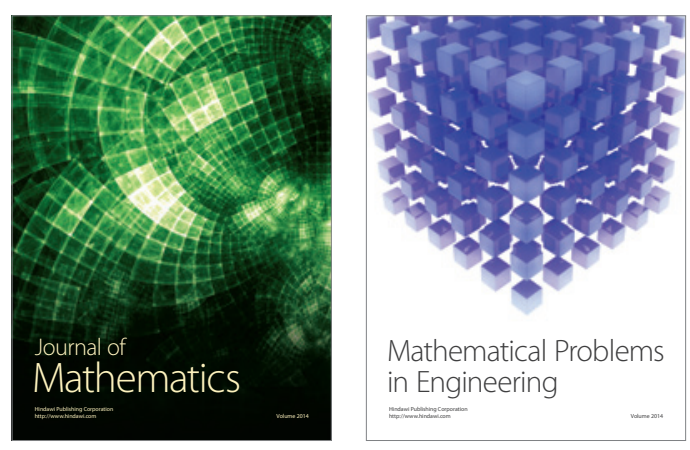

Mathematical Problems in Engineering
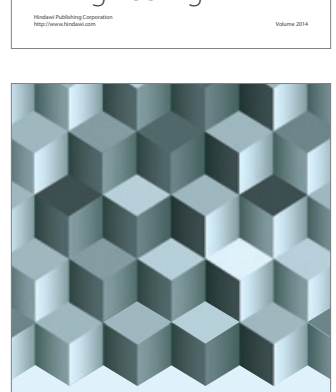

Journal of

Function Spaces
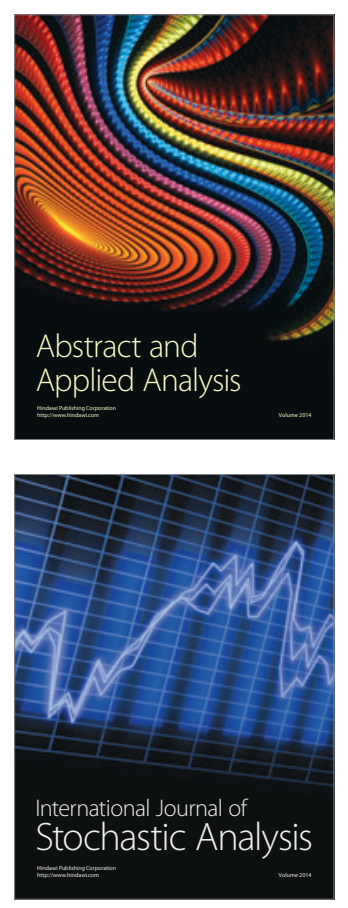

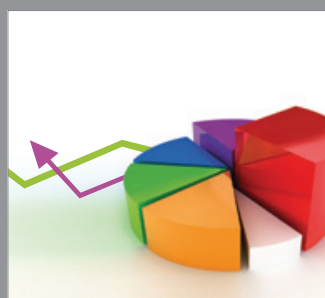

ournal of

Probability and Statistics

Promensencen
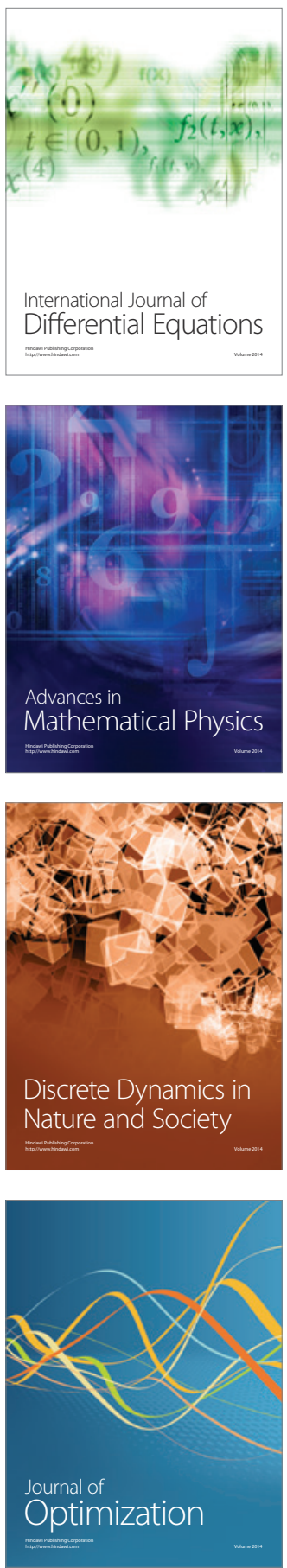